\title{
THE EFFECT OF DIFFERENT MODEL TECHNIQUES ON THE MARGINAL GAP OF IMPLANT SUPPORTED FIXED PARTIAL DENTURE
}

\author{
Maged Zohdy* and Andy Gabra**
}

\begin{abstract}
Statement of problem: The conventional model fabrication methods have multiple errorintroducing steps such as impression, stone setting, waxing and casting; therefore a more reliable and quality controlled method of model fabrication is required

Objective: This research was designed to evaluate the effect of different model fabrication techniques: Conventional stone cast, Rapid Prototyping-3D printing and CAD/CAM on the marginal gap of implant supported fixed partial denture.

Materials and methods: An epoxy resin cast simulating a clinical case with missing lower first mandibular molar was fabricated that served as a master cast from which an optical impression was done to fabricate a 3D printed model and a milled model , and another rubber base impression was done to fabricate a conventional cast, upon these three casts, five three units bridge was fabricated on each model, then marginal gap of implant supported fixed partial denture was evaluated using stereomicroscope.
\end{abstract}

Results: Two-way ANOVA revealed significant differences were found between the model manufacturing techniques. 3D-printing showed the lowest marginal gap value among all groups.

\section{INTRODUCTION}

Computer aided design and manufacture $(\mathrm{CAD} /$ CAM) techniques have been successfully introduced in the field of fixed partial dentures ${ }^{(1,2)}$. Nowadays different model cast techniques are applied, from conventional stone casts and milled CAD/
CAM casts and lately 3D printed casts have been introduced. This development was supported by the capability of modern imaging modalities, like spiral CT and MRI, to produce continuous volumetric data sets, which provide the input data for model generation.

\footnotetext{
* Associate professor of fixed prosthodontics ain shams university and british university

** Lecturer of fixed prosthodontics ain shams university
} 
However the literature on applications of Rapid Prototyping technologies in the dental areas still rare even though more and more attention has been paid to this area. Currently, most dental jobs are still being performed by dental technicians manually.

The use of dental implants to replace natural teeth has become common place in contemporary restorative and surgical dental practices throughout the world, it became the treatment of choice in many if not in most clinical situations. Their efficacy has been well documented in the dental literature. There have been many advances in surgical techniques and implant design features, and the use of implants in edentulous sites can be successful and can have predictable, functional, and esthetic outcomes. ${ }^{(3)}$

Many studies investigated the factors determining superstructure accuracy, mainly impression and definitive cast accuracy, accuracy of laboratories processes and the component machining tolerances provided by the manufacturer. Tan ${ }^{(4)}$ pointed out that each single clinical and laboratory stage contributes to the inaccuracy level of a bridge.

\section{Impression technique}

$\boldsymbol{C a r r}^{(5)}$ compared a direct and an indirect impression technique for a 5- Implant model and concluded that the direct transfer method produced a more accurate cast.

Assif et al $^{(6)}$ Reported that using acrylic resin to splint transfer copings in the impression material produced more accurate results than splinting the transfer copings directly to the acrylic resin custom tray or leaving the transfer coping unsplinted. Assif et al reported that splinting pick up type impressions copings during the impression phase would yield better results.

\section{Master cast accuracy}

Master cast accuracy has been extensively studied, when conventional crown and fixed partial denture techniques for the fabrication of the implant supported fixed prosthesis are used, the restoration are fabricated on master cast in the laboratory where die stone are the most commonly used die material. Expansion of the die stone occurs on setting of the fixed prosthesis ${ }^{(7,8,9)}$

When an in vitro replica of the mouth was evaluated with spans of $50 \mathrm{~mm}$ across the arch in the molar area and $20 \mathrm{~mm}$ across the canine region, it was reported that the solid cast poured in die stone produced cross arch discrepancies of $201 \mu \mathrm{m}$ between the posterior abutments and $77 \mu \mathrm{m}$ between the anterior abutments when compared with the replica, Hence it can be concluded that die stone expansion results in inaccuracies in the master casts when compared with the original patient replicas. On the other hand

\section{Fabrication method}

Another point of interest is the influence of different fabrication methods on the accuracy of the bridge. Carr et $\boldsymbol{a l}^{(10)}$ found that prefabricated gold cylinders would be advantageous for fabricating screw-retained bridges,

Though he did not find any significant difference in using prefabricated gold components when compared with conventionally used plastic copings.

Computer-aided design and manufacture (CAD/ CAM) techniques have been successfully introduced in the field of fixed partial dentures ${ }^{(1)(11)}$ over a number of years. Computer-assisted design and manufacturing (CAD/CAM) technology was introduced in Europe in 1980s for rapid generation of the prostheses made of machinable ceramics. During the last two decades, dental CAD/CAM technology has been used to replace the laborious and timeconsuming, conventional lost-wax technique. CAD/ CAM technology has revolutionized dentistry. Restorations can be produced much more quickly and efficiently, eliminating the need for temporary restorations. Moreover, with the computer controlled design and manufacturing, making prostheses with consistent quality become possible..$^{(12)}$ 


\section{Production of models through CAD/CAM versus Conventionally fabricated models}

Dental CAD/CAM technology consists of digital image generation and data acquisition (Scanner), computer-assisted milling systems and tooling systems. The scanner scans the dental preparation either intraorally or extraorally through scanning a laser visible stone casted model of the preparation. Various methods of scanning are used throughout dentistry, including laser and lined light. ${ }^{(13,14)}$ The scanner can only collect data on surfaces within the line of sight. Areas on the surface that are obscured or at too great an angle to the line of sight will not appear in the scan data. Moving either the object or the scanner and repeating the process overcomes this problem. For scanning a cast of a patient, several scans may be necessary to create a point cloud. Computer software is then used to create Surfaces from these points. This method creates the surface of a 3D object by approximating the point cloud with a series of connected triangular facets. These triangular faceted models, termed a stereolithography or STL file, are commonly used in transferring CAD models where milling can be performed using a milling machine to produce the dental cast.

On the contrary the conventional cast is made using clinical and laboratory steps where every step might incorporate some errors in the model final production accuracy, starting with the impression material properties and dimensional changes through the dental stone proportioning and mixing and ending with model expansion. Other deficiencies arise from difficulties in their storage and retrieval due to their physical bulk.

\section{Advantages of using CAD/CAM}

The exponential increase in the application of computer-aided manufacturing in prosthodontics is attributed to continuous systems development and refinement, greater ability for quality control, parallel material development, and the possibility of virtual evaluation. Other areas of refinement include scanning technology, modeling software, and production systems, and the systems are becoming more user friendly. ${ }^{(15)}$

\section{a) Additive Manufacturing}

Additive manufacturing systems have recently been introduced as a method to construct dental restorations and medical devices usually layer upon layer.(16) Additive manufacturing is defined by the American Society for Testing and Materials (ASTM) as:

The process of joining materials to make objects from $3 D$ model data, usually layer upon layer, as opposed to subtractive manufacturing methodologies.

Additive manufacturing technology has attracted enormous interest among researchers because it greatly facilitates the realization of three-dimensional (3D) objects. Common additive technologies in medicine are Selective Laser Sintering, Fused Deposition Modeling, Multi-Jet Modeling and Stereo-lithography. Here the model is built layer by layer according to 3D contour data. In comparison to subtractive techniques, the additive technologies can produce arbitrarily complex structures and cavities. ${ }^{(17)}$

\section{Advantages and disadvantages of Additive man- ufacturing}

Among the advantages of additive manufacturing is the ability to produce customized work pieces that fit patient hard and/or soft tissues(18). The work pieces can include detailed morphology, sharp corners, undercuts, or voids. Such features may be desirable for facial prostheses. Because no drilling tool is involved, no compensation feature is required as is necessary for the subtractive manufacturing. Further, the whole production process is passive and involves no force application. However, due to the production procedure, which involves sequential layering, the external surface tends to have stepped and coarse morphology representing each fabrication layer along the construction direction. 


\section{b) Subtractive Manufacturing}

If we look at where we are today then in fact $\mathrm{CAD} / \mathrm{CAM}$ in dentistry is primarily based upon the idea of subtractive manufacturing. Subtractive technique used for medical applications is milling, which was derived from numerically controlled (NC) machine processing. In this case, the shape of the model will be milled from a block. In this process, a power driven machine tools and drills are used with sharp cutting tools to mechanically cut the material to achieve the desired geometry with all steps controlled by a computer program. Thus, one starts out with a block of the desired material and the machine cuts away the bits that are not wanted. The advantages are the low material costs and the possibility that these models can be worked on with surgical instruments.(19)

\section{Advantages and disadvantages of Subtractive manufacturing:}

As stated earlier, milling is anticipated to eliminate waxing, investing, and casting of prostheses, which is assumed to improve the overall precision. However, there is a lack of compelling evidence supporting this assumption for tooth-supported restorations(20), because, in terms of fit, there is an overall tendency for the restorations produced by conventional methods to exhibit better fit than milled restorations. This applies to milled metal ${ }^{(21)}$ and ceramic restorations. ${ }^{(22)}$

\section{Margin adaptation}

One of the most important criteria for the longterm success of ceramic restorations is their marginal adaptation i.e. the distance between the finish line and the restoration margin ${ }^{[23]}$. It is necessary to minimize the marginal gap, since a significant space between the tooth and the restoration exposes the luting material to the oral environment, thus resulting in a more aggressive rate of cement dissolution caused by oral fluids and chemomechanical forces ${ }^{[24]}$. The consequent microleakage may result in in- flammation of the periodontal tissues, and subsequent failure of the prosthesis ${ }^{[25,26]}$.

Several methods for studying marginal adaptation have been proposed in the literature. Among them, Holmes et al in $1989{ }^{[27]}$ established a uniform vocabulary for referring to the characteristics under study: internal opening, marginal opening, horizontal and vertical marginal discrepancy, short and over marginal extension, marginal absolute discrepancy and settlement discrepancy. Other studies on marginal fit ${ }^{[28,29]}$, detail their evaluations in different regions: labial, mesial, distal and lingual.

\section{MATERIALS AND METHODS}

A cast simulating a clinical case with missing lower first mandibular molar was fabricated from epoxy resin that will serve as a master cast from which an optical impression will be done to fabricate a 3D printed model and a milled model , and another rubber base impression will be done to fabricate a conventional cast, upon these three casts, five three units bridge will be fabricated on each model to assess the marginal gap distance.

\section{Master model Construction:}

A one step addition silicone impression was taken for a clinical case having 2 implants placed, The first one in the lower second premolar region and the second implant in the lower second molar region after screwing the implant abutment to the implants, after complete setting of the impression material, the abutments were unscrewed from the implants and attached to the corresponding analogues diameters and seated in place in the impression. Then the impression was boxed. Epoxy resin base and catalyst were then proportioned according to the manufacturer instructions in a glass container and mixed thoroughly using a glass rod on top of a vibrator. The epoxy resin was then left aside for ten minutes to allow escaping of air bubbles and then was poured slowly in the impression and around 
the implant analogues and left to set in place ( Initial setting took about 24 hours, while final setting took around 3 days). Slight trimming of the model was made after final setting of the epoxy resin cast (Fig.1).

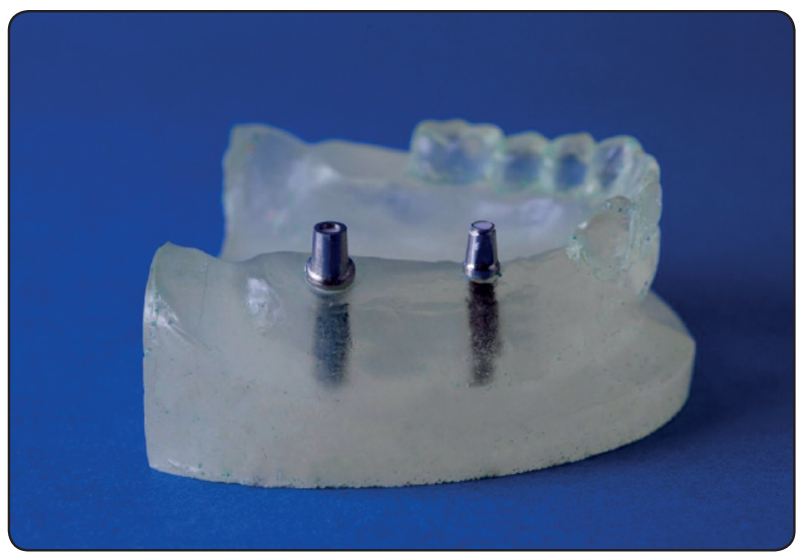

Fig. (1) Epoxy resin cast

A total of fifteen fixed-fixed bridges were constructed in this study $(n=5)$, divided into three groups according to the model technique as follows

Group A: Three units fixed-fixed zirconia bridge fabricated using rapid prototyping cast

Group B: Three units fixed-fixed zirconia bridge fabricated using a conventional stone cast made using an addition silicone impression.

Group C: Three units fixed-fixed zirconia bridge fabricated using a milled cast

\section{Group (A) Rapid Prototyping cast construction:}

In this group a 3D printed cast was made by scanning the epoxy cast using 3Shape desktop (3Shape ${ }^{\circledR}$ dental Denmark, Fig:8) scanner and generating an .STL file where it can be directly sent to the $3 \mathrm{D}$ printing machine, Some editing in the. $\mathrm{STL}$ file was made including digital trimming of the cast base.

Using additive manufacturing device; the Envision TEC (Ferndale, MI) Perfactory $\left.{ }^{\circledR}, 10\right)$ which is based on the idea of Continuous Digital Light projection (cDLP) which utilizes a DLP® (Texas Instruments, Dallas, TX) chip to print the cast layer by layer utilizing the projection of a UV light to polymerize the layers until the whole cast is printed starting with the base and ending with teeth and abutments. The raw material used in in production of the printed item is a Photopolymer which in fact is a Mixture of acrylic acid esters and photoinitiator (Fig:2).

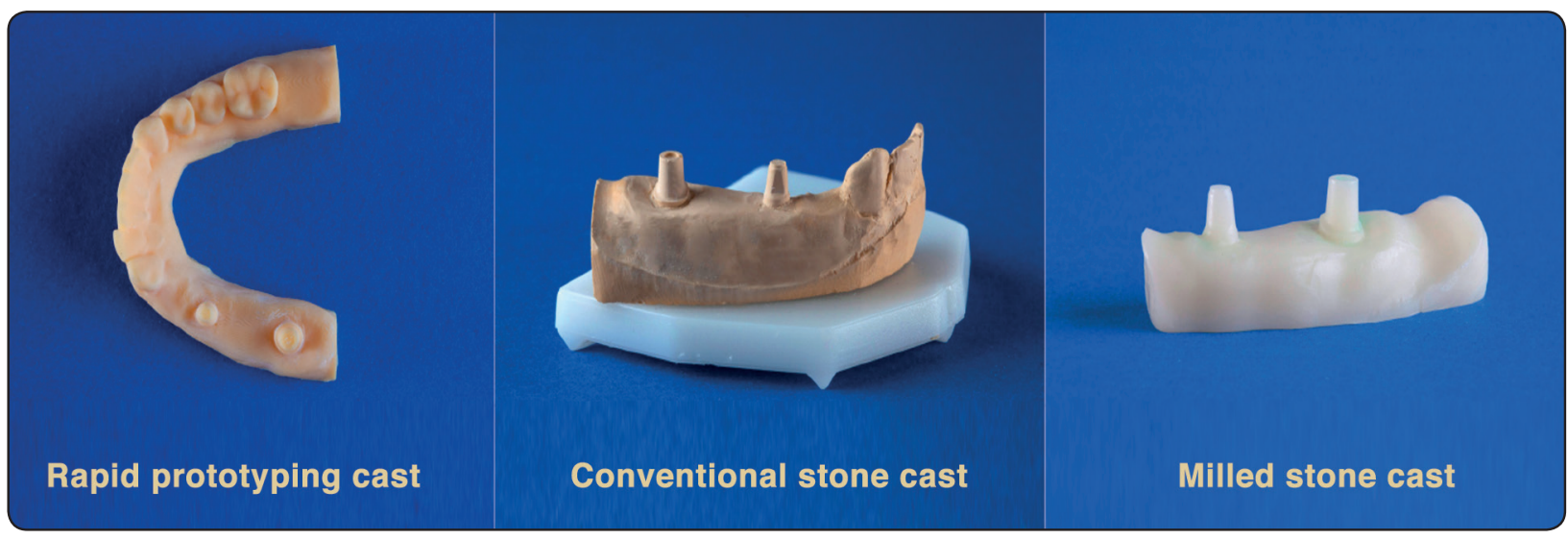

Fig. (2) 


\section{Group (B) Model construction:}

A One-step impression technique was made for the epoxy cast, by mixing an equal amount of $\mathrm{Pa}$ nasil soft (Kettenbach Gmbh \& Co) putty base and catalyst and injecting Identium (Kettenbach Gmbh \& Co) light body around the abutments, after complete setting of the impression material the impression was boxed using pink wax. Dental stone type IV (Fuji GC USA) was proportioned according to the manufacturer's recommendation and mixed using a vacuum mixing machine, poured slowly in the impression on top of a vibrator to allow for the escape of the air bubbles and prevent their entrapment. The stone was allowed to set for 45 minutes, removed from the impression, trimmed and inspected for air bubbles(Fig:3)

\section{Group (C) model construction:}

Group C model was made using subtractive manufacturing technique, The epoxy cast was scanned using 3Shape desktop (3Shape ${ }^{\circledR}$ dental Denmark) ,scanner to generate an.STL file where it can be directly sent to the milling machine, Some editing in the .STL file was made including digital trimming of the cast base. The cast was milled using Zirkonzahn M5 (ZirkonZahn Italy) using a PMMA blank and grinding burs of $2 \mathrm{~L}, 1 \mathrm{~L}$ and $0.5 \mathrm{~L}$ (Fig:4)

\section{Construction of the Zirconia monolithic bridges}

After fabricating the 3 types of casts (conventional, 3D printed and milled cast) from the same epoxy master cast, each cast was scanned using Identica Blue desktop scanner (Medit Co Ltd Korea) to design the bridges, designing was done Using Exocad software version 2014.02 (Dental DB-Medit.) in windows 7 ultimate which is a 3 dimensional software that features a menu controlled user interface that provide full capabilities for the operator to scan the preparation and design the required restorations through a step by step guidance.

\section{Milling of the bridges}

The bridges of group A,B and C was milled using VHF CAM 5-S1 impression machine through Dental CAM software v6.05.17 Beta

The .STL file obtained from the exocad was imported and the material was selected in the dialogue box to Zirconia blanks (BruxZir translucent Zirconia milling blanks Prismatik dental craft, Inc Irvine CA Glidewell)

After the bridges were milled the connecting sprue were cut using a straight hand piece and a mounted fissure bur, finishing of the bridges and smoothening of the sprue are done. All bridges were ultrasonic cleaned for 30 seconds in an ultrasonic cleaning machine filled with fresh distilled water to remove all residual zirconia dust, and then the bridges were left to dry for 2 minutes under a drying heat convection lamp.

\section{Sintering of Zirconia bridges}

As the bridges were milled in pre sintered state, they underwent a sintering cycle, All bridges were placed in the sintering tray containing appropriate sized zirconia beads, the sintering firing cycle was carried out in Nabertherm (Bahnhofstrasse 20 Lilienthal/Germany) furnace

\section{Checking and verification}

After finishing the sintering cycle, the bridges were checked on the corresponding models. All bridges were checked for the fitting surfaces, seating, adaptation and margins, where group A where checked on the 3D printed model cast, group $\mathbf{B}$ were checked on the stone cast and group $\mathbf{C}$ were checked on the milled casts.

\section{Margin adaptation measuring}

Then morphometric measurements were done on an IBM compatible personal computer equipped with the Image-tool software (Image J 1.43U, National Institute of Health, USA). which was used 
for image analysis. Within the Image $\mathbf{J}$ software, all limits, sizes, frames and measured parameters are expressed in pixels. Therefore, system calibration was done to convert the pixels into absolute real world units. Calibration was made by comparing an object of known size (a ruler in this study) with a scale generated by the Image $\mathbf{J}$ software. The vertical gap distance was measured for each shot [ 5 equidistant landmarks along the cervical circumference for each surface of the bridge (Fig.5) (Mesial, buccal, distal, and lingual).

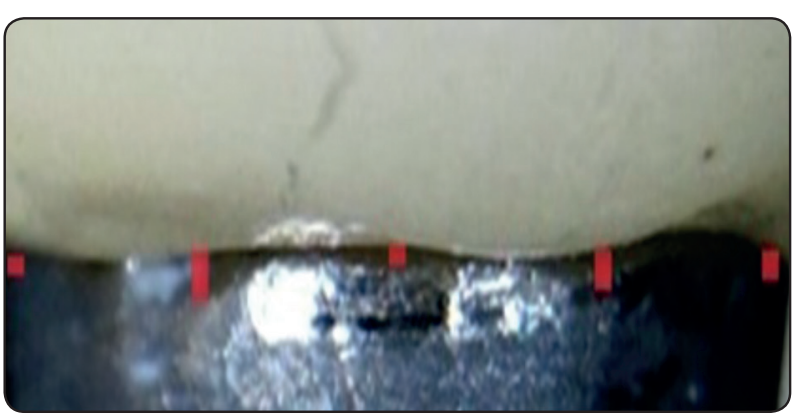

Fig. (5) Stereomicrograph with lines of measurements at equidistant points.
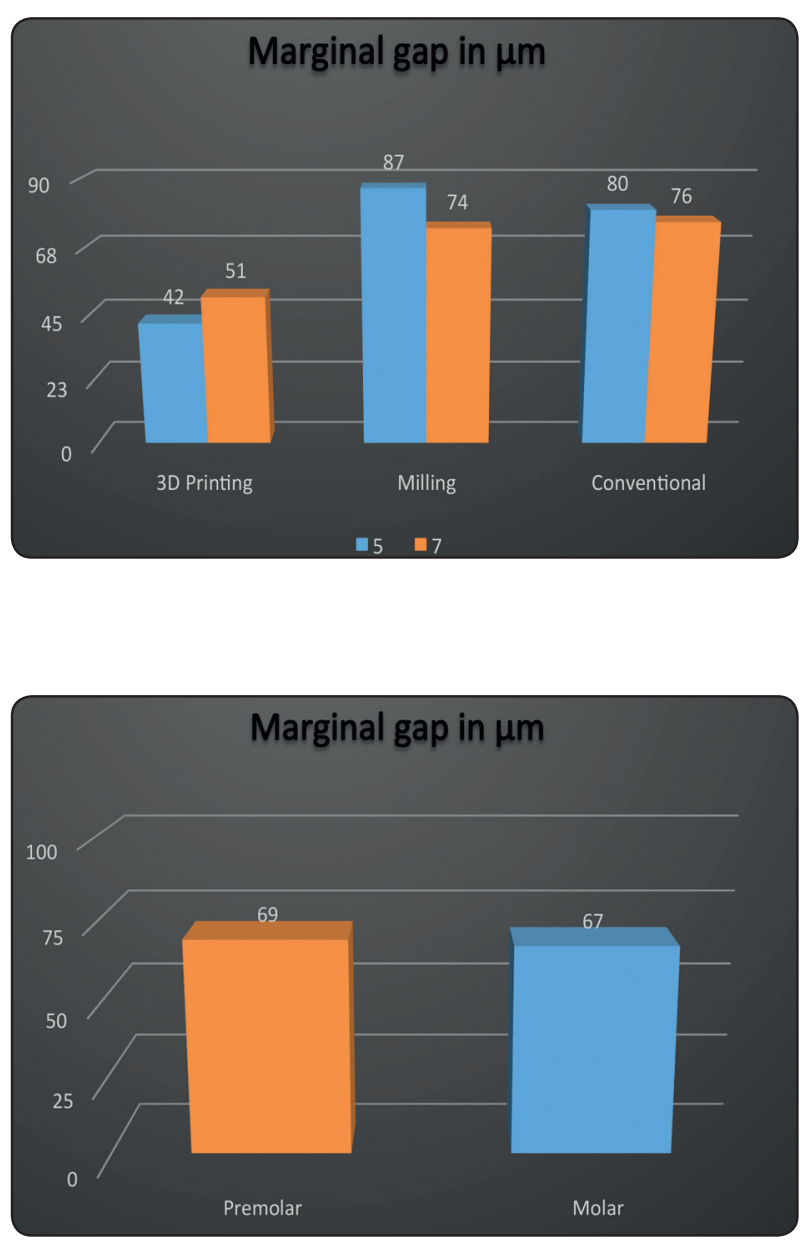

Means with different superscript letters are statistically significant $p<0.05$

\section{RESULTS}

Data was checked for normal distribution and analyzed using two-way analysis of variance. Twoway ANOVA revealed significant difference between the subgroups. Significant differences were found between the model manufacturing techniques. The milling and the conventional techniques showed significantly higher marginal gap when compared to the $3 \mathrm{D}$ printing.

\begin{tabular}{|c|c|c|}
\hline & 5 (Premolar) & 7 (Molar) \\
\hline 3D Printing & $42(5)^{\mathrm{a}}$ & $51(5)^{\mathrm{a}}$ \\
\hline Milling & $87(7)^{\mathrm{b}}$ & $74(4)^{\mathrm{b}}$ \\
\hline Conventional & $80(6)^{\mathrm{b}}$ & $76(5)^{\mathrm{b}}$ \\
\hline
\end{tabular}

\section{DISCUSSION}

This research subject was chosen due to the claimed importance of passivity of fit on the longterm success of implant supported fixed prosthesis. It was carried out to evaluate the effect of different model techniques on the passivity of fit of implant supported fixed prosthesis.

Traditional conventional cast is fabricated using clinical and laboratory steps that might increase errors in the model final production accuracy as impression material properties, dimensional changes, dental stone proportioning and mixing and model expansion. 
Rapid Prototyping technology has attracted enormous interest among researchers because it greatly facilitates the realization of bespoke threedimensional 3D objects.

To simulate a clinical case; a secondary impression was taken from a clinical case with a missing lower first molar and a standard abutments of size $3.5 \mathrm{~mm}$ and $5 \mathrm{~mm}$ placed on the respective implants. After the impression was set the abutments were unscrewed and attached to the respective analogues and placed in the impression for epoxy pouring step. The analogues were about $14 \mathrm{~mm}$ apart, which is the average distance between lower second premolar and second molar. ${ }^{(30)}$

The model was constructed from epoxy resin (Chemapoxy, CMB, Egypt) which has a modulus of elasticity similar to that of jaw bones ${ }^{(31)(32)}$,

After setting of the epoxy master cast, The different models were fabricated either using an optical impression as in case of 3D printed cast and milled cast, or from a conventional impression and pouring dental stone type IV

The two newly introduced model materials were compared to the conventional cast. The first model was fabricated using 3D printed technology (EnvisionTEC Ferndale, MI Perfactory ${ }^{\circledR}$ ), the second model was fabricated using milling technique (Zirkonzahn M5 ZirkonZahn Italy) using a PMMA blank, both using an optical impressions to assess the additive, subtractive in comparison to conventional technique.

The measurement model used was the master epoxy cast ${ }^{(30)}$. on which the optical/conventional impressions was done on, The bridges were constructed on their master cast after scanning of the models using Identica Blue desktop scanner (Medit Co Ltd Korea) .

Standardization of the bridges tested for passivity of fit was done using milled full contour monolithic Zirconia bridges using the same design (BruxZir Zirconia blanks Glidewell, USA) using Exocad software version 2014.02 (Dental DB-Medit.) in windows7.

The purpose of using a full contour monolithic Zirconia is the elimination of the veneering factors that might affect the standardization, as it was stated earlier that there is a significant increase in strain development after ceramic veneering in the conventionally fabricated screw-retained fixed partial dentures. ${ }^{(31)}$

\section{Using light microscope for measuring margin adaptation}

The vertical cervical marginal gap measurement was selected as the most frequently used to quantify the accuracy of fit of a restoration. ${ }^{[33]}$

The assessment of the marginal adaptation of the crowns was performed using stereomicroscope; all measurements were made by the same operator to avoid error.

Abhishek et al in $2011{ }^{[34]}$ indicate that the most commonly used method i.e. explorer, for evaluating the fit of castings may be even less reliable than reported. When margins are evaluated with an explorer acceptance is more likely to be based on the size and character of overhangs and ledges than on the actual size of opening of the margins

The sensitivity of explorer and elastomeric disclosing media as compared to stereomicroscope at $30 \mu \mathrm{m}$ was $39 \%$ and $10 \%$ respectively. Higher values of sensitivity for explorer technique as compared to elastomeric disclosing media indicate that explorer technique is more reliable as compared to elastomeric disclosing media in detecting marginal gaps

However values of sensitivity for both the techniques evaluated is considerably low, indicating that these techniques may not be adequate to determine presence of marginal gaps of or less than $30 \mu \mathrm{m}^{[34]}$

A recent study has reported that clinical detection of marginal gap size with similar sensitivity and specificity as stereomicroscope occurs at greater 
than or equal to $124 \mu \mathrm{m}^{[35]}$. Although the stereomicroscope cannot be used to assess restorations intra-orally, the future development of an intraoral apparatus may be of value. However, the use of a stereomicroscope as a supplement method for assessing castings on dies may provide a higher degree of marginal gap detection prior to examination of these castings intra-orally ${ }^{[35]}$

Though very important element in evaluating a restoration; there is no agreement in definition of a clinically acceptable margin ${ }^{[36]}$.

In this study, the conventional cast showed the highest values of margin gap followed by the milled cast and then the $3 \mathrm{D}$ printed cast, This finding might be associated with conventional cast fabrication and pouring technique sensitivity, and in milled cast fabrication by grinding burs or milling axes, might be the cause of increased strain in the overall channel comparison.

In support of this results Karl M. et al in 2012 found that intraoral digitization of dental implants appear to be at least as precise as conventional impression taking and master cast fabrication ${ }^{(37)}$

The bridges fabricated on the stereolithography 3D printed model showed the lowest amount of strains might be attributed to the fact that the 3D printed models exhibits no or nil amount of internal stresses due to the mode of fabrication through building the cast layer by layer rather than grinding as in milled cast or by a chemical reaction that involves expansion or contraction as in case of conventionally fabricated casts. ${ }^{(38)}$

This study showed that using digital virtual impression, 3D printing and milling casts are as precise or better than conventional impression and casts pouring, potential fabrication inaccuracies due to processing of Zirconia ceramic may have counteracted the greater precision resulting from digital impressions. ${ }^{(39)}$

However the literature on applications of Rapid Prototyping technologies in the dental areas still rare even though more and more attention has been paid to this area. Currently, most dental jobs are still being performed by dental technicians manually.

\section{CONCLUSION}

In this study it was concluded that virtual impression, 3D printing and milling casts are as precise or better than conventional impression.

Within the limitations of this in vitro study, it was possible to conclude that:

1) $3 \mathrm{D}$ printed casts have the lowest marginal gap values

2) Conventional stone casts showed the highest marginal gap

\section{Clinical recommendation:}

3D printed casts can be used in fixed partial dentures with minimal marginal gap.

\section{REFERENCES}

1. Rekow D, Computer-aided design and manufacturing in dentistry: a review of the state of the art. J Prosthet Dent 1987;58:512-6.

2. Duret F, Preston J, Duret B. Performance of CAD/CAM crown restorations. J Calif Dent Assoc. 1996;9:64-71.

3. Bothe RT BLDH. Reaction of bone to multiple mettalic implants. Surg Gynecol Obstet 1940;(71):598-602.

4. Tan KB, Rubenstein JE. Three dimensional analysis of the casting accuracy of one-piece, osseointegrated implantretained prostheses. Int J Prosthodont 1993 Jul-1993 Aug;6(4):346-63.

5. Carr AB.Comparison of an impression technique for a five implant model. Inte J of Oral and maxilofacial. 1991 Winter 6:(4);448-55.

6. Assif D, Fenton A, Zarb G, Schmidt A. Comparative accuracy of implant impression procedures. Int J Periodontics Restorative Dent 1992;12:112-121.

7. Wise M. Fit of implant supported fixed prosthesis fabricated on master casts made from a dental stone and adental plaster . J Prosthet Dent 2001 Nov;86(5):532-8.

8. Millstein PL. Determining the accuracy of gypsum casts made from ype VI dental stone. J Oral Rehabil 1992 May;19(3):239-43. 
9. Vigolo P, Millstein PL. Evaluation of master cast techniques for multiple abutment implant prostheses. Int J Oral Maxillofac Implants 1993;8:439-46.

10. Carr AB, Brunski JB. Effects of fabrication, finishing and polishing procedures on preload in prosthesis using conventional gold and plastic cylinders. Int J Oral Implats 1996 Sep11(5)589-98.

11. Duret F, Preston J, Duret B. Performance of CAD/CAM crown restorations. J Calif Dent Assoc 1996;9:64-71.

12. E.D. Rekow, A.G. Erdman, D.R. Riley, B. Klamecki, CAD/CAM for dental restorations-some of the current challenges, IEEE Trans, Biomedical Engineering 38 (4) (1991) 318-414. .

13. Brosky ME, Pesun IJ, Lowder PD, DeLong R, Hodges JS. Laser digitisation of casts to determine the effect of tray selection and cast formation technique on accuracy. J Prosthet Dent 2002;87:204-9.

14. Sohmura T, Kojima T, Wakabayashi K, Takahashi J. Use of an ultrahigh speed laser scanner for constructing threedimensional shapes of dentition and occlusion. J Prosthet Dent 2000;84:345-52.

15. F. Beuer, J. Schweiger, and D. Edelhoff, "Digital dentistry: an overview of recent developments CAD generated res torations,"BritishDentalJournal,vol.204,no.9,pp.505-511, 2008.

16. P. A. Webb, "A review of rapid prototyping (RP) techniques in the medical and biomedical sector," Journal of Medical EngineeringandTechnology,vol.24,no.4,pp.149-153,2000.

17. Zonneveld FW, van der Dussen MFN. Three-dimensional imaging and model fabrication in oral and craniofacial surgery. Oral Maxillofacial Surg Clin N Am 1992;4:19-33.

18. B. K. Davis, "The role of technology in facial prosthetics," Current Opinionin Otolaryngology and Headand Neck Surgery, vol.18,no.4,pp.332-340,2010. .

19. Van Roekel NB. Electrical discharge machining in Dentistry. Int J Prosthodont 1992;5:114-21.

20. P. L. Tan, D. G. Gratton, A. M. Diaz-Arnold, and D. C. Holmes, "An in vitro comparison of vertical marginal gaps of CAD/CAM titanium and conventional cast restorations," JournalofProsthodontics,vol.17,no.5,pp.378-383,2008. .

21. H. S. Han, H. S. Yang, H. P. Lim, and Y. J. Park, "Marginal accuracy and internal fit of machine-milled and cast titanium crowns,'The Journal of ProstheticDentistry, vol. 106, no. 3, pp. 191-197,2011. .

22. Aboushelib M.N, W. A. Elmahy, and M. H. Ghazy, "Internal adaptation,marginalaccuracyandmicroleakageofapressable versus a machinable ceramic laminate veneers," Journal of Dentistry,vol.40,pp.670-677,2012. .

23. Glossary of Prosthodontic Terms. J Prosthet Dent 2005; 94: 92.

24. Jacobs MS, Windeler AS. An investigation of dental luting cement solubility as a function of the marginal gap. J Prosthet Dent 1991; 65:436-42.

25. Knoernschild KL, Campbell SD. Periodontal tissue responses after insertion of artificial crowns and fixed partial dentures. J Prosthet Dent 2000;84:492-8.

26. Sailer I, Feher A, Filser F, Gauckler LJ, Luthy H, Hammerle $\mathrm{CH}$. Five-year clinical results of zirconia frameworks for posterior fixed partial dentures. Int J Prosthodont 2007; 20:383-8.

27. Holmes JR, Bayne SC, Holland GA, Sulik WD. Considerations in measurement of marginal fit. J Prosthet Dent 1989; 62: 405-408.

28. Groten M, Axmann D, Pröbster L, Weber H. Determination of the minimum number of marginal gap measurements required for practical in-vitro testing. J Prosthet Dent 2000;83:40-9.

29. In-Sung Yeo, Jae-Ho Yang. In vitro marginal fit of three all ceramic crown systems. J Prosthet Dent 2003; 90: 405-408.

30. Michalakis K, Pissiotis AL, Kang K. The effect of thermal cycling and air abrasion on cement failure loads of 4 provisional luting agents used for the cementation of implant supported fixed partial denture. Int J Oral Maxillofac Implants 2007 Aug:22(4):517.

31. Karl M, Rosch S, Graef F, Taylor TD, Heckmann SM. Static implant loading caused by as-cast metal and ceramic-veneered superstructures. J Prosthet Dent 2005;93:32430.

32. Keavany TM, Guo XE, Wchtel EF. Trabecular bone exhibits fully linear elastic behaviour and yields at low strains. J Biomech 1994 Sep;27(9):1127-36.

33. Wolfart S, Wegner SM, Al-Halabi A, Kern M. Clinical evaluation of marginal fit of a new experimental all-ceramic system before and after cementation. Int $\mathrm{J}$ Prosthodont 2003;16:587-92. 
34. AbhishekR,Vikas K, Comparative analysis of the clinical techniques used in evaluation of marginal accuracy of cast restoration using stereomicroscopy as gold standard, J AdvProsthodont 2011;3:69-75.

35. Jahangiri L, Wahlers C, Hittelman E, and Matheson P.Assessment of sensitivity and specificity of clinical evaluation of cast restoration marginal accuracy compared to stereomicroscopy. J Prosthet Dent 2005;93:138-42.

36. Hunter A.J, and Hunter A.R. Gingival margins for crowns: A review and discussion. Part II: Discrepancies and configurations. J Prosthet Dent 1990; 64:636-642.
37. Karl M, Wichmann MG, Heckmann SM. Strain development in 3 unit implant supported CAD/CAM restorations. Int J Oral Maxillofac Implants 2008;23:648-652.

38. Gomez EA, Assuncao WG, Tabata LF. Effect of passive fit absence in the prosthesis/implant/retaining screw system"A two dimensional finite element analysis. J Craniofac Surg 2009;20:2000-2005.

39. Abduo J, Lyons K, "Rationale for the use of CAD/CAM te chnologyinimplantprosthodontics,'InternationalJournalof Dentistry,vol.2013,ArticleID768121,8pages,2013. 